American Journal of Animal and Veterinary Sciences 7 (2): 75-79, 2012

ISSN 1557-4555

(C) 2012 Science Publications

\title{
Production of Male-Sterility Mouse by Resection of the Tail Epididymis
}

\author{
Xianju Huang, Xuguang Wang, Xueshan Ma, Nazim Ally and Honglin Liu \\ College of Animal Science and Technology, Nanjing Agricultural University, China
}

\begin{abstract}
Problem statement: Sterile males are bred with females to produce pseudopregnant recipients for oviduct and uterine transfers. Therefore, to make a sterile male mouse maybe the fist and fundamental procedure for embryo transfer. However, when people produce a sterile male mouse always abandon the sperm of the excellent male mouse. Approach: Here, the authrous present an efficient and simple procedure utilizing specific methods that make a sterile male mouse by resection of the caudal epididymis meanwhile collect sperm from the caudal epididymis for in vitro fertilization and then cauterize the vestigial epididymis to produce sterile male mouse. The experimental males and females are all normal mouse. Results: (1) The in vitro fertilization to cater to the demand of the mount of embryo for oviduct and uterine transfers and the sterile mouse are prepare for mate with female to produce pseudopregnant recipients for oviduct and uterine transfers; (2) Accessing the caudal epididymis through the scrotal sac has less invasive with mouse. Conclusion/Recommendations: Ablating the caudal epididymis of male mice has not reduced the achievement rate of copulation and reconversion. In vitro fertilization to harvester embryo can use for embryo and other preparing experiment.
\end{abstract}

Key words: Sterile male mouse, the caudal epididymis

\section{INTRODUCTION}

The rapid increase in the development of pseudopregnant recipients' mouse for oviduct and uterine transfers have resulted in growing demand for sterile mice. Accordingly, we developed a new procedure of makeing sterile mice with less work and created a precise and less invasive method.

Two methods of making sterile male mouse have been reported (Nagy, 2003): one is by accessing the vas deferences through the abdomen; the other is less invasive method by accessing the vas deferences through the scrotal sac. Here we presented the progress to make male-sterility mouse by resection the caudal epididymis. While a laboratory works demand mouse embryo transplant, there is need for pseudopregnant recipients' female mouse in case of oviduct and uterine transfers, there may also require large number of mouse embryo by in vitro fertilization. Epididymis is the mature sperm library of male mouse and is just the position to collect sperm for in vitro fertilization, while cut or cauterized epididymis may prevent the mature sperm transportation and result in male mouse sterility. Here we combine the two mechanisms: acquisition the sperm for in vitro fertilization and make sterile male mouse .Our work provided a system that save time, excellent male mouse for experiment and always produces excellent male-sterility mouse.

\section{MATERIALS AND METHODS}

All mice were wild type (wt) at 8-12 weeks old. Male mice were all housed in one cage. Before the experiment, we had superovulate the wt female mouse at 5:00 am and $48 \mathrm{~h}$ later each male mouse was introduced to one female mouse. Until the second morning the females were checked for the presence of vaginal plugs, an indication that sexual activity had taken place. If a vaginal plug was present (Daniel, 1978), the female was placed in a separate cage, this was repeated ten times for four days until ten best female mouse was selected.

Resection of the caudal epididymis to make a sterile male mouse is modified from both the conventional methods and procedures. First, weigh and anaesthetized the experimental male mouse by Intraperitoneal (IP) injection of tribromoethanol, avertin $0.02 \mathrm{~mL} \mathrm{~g}^{-1}, 1.25 \%$ (volume ratio) (Papaioannou et al., 1993; Erhardt et al., 1984; Nowrouzian et al., 1981; Zeller et al., 1998). 
American J. Animal \& Vet. Sci., 7 (2): 75-79, 2012

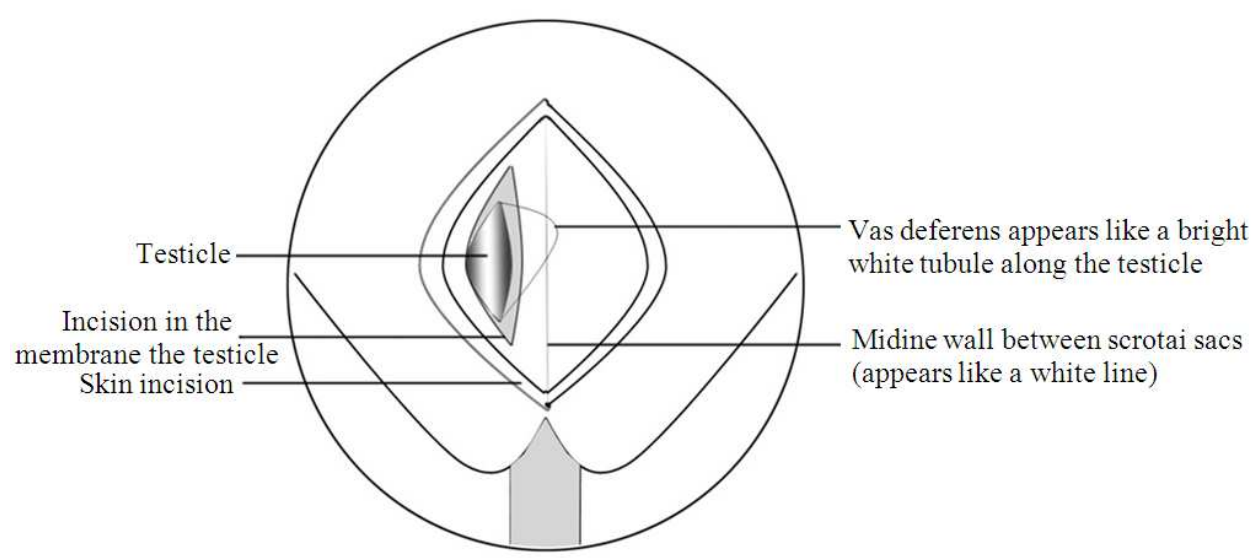

(A)

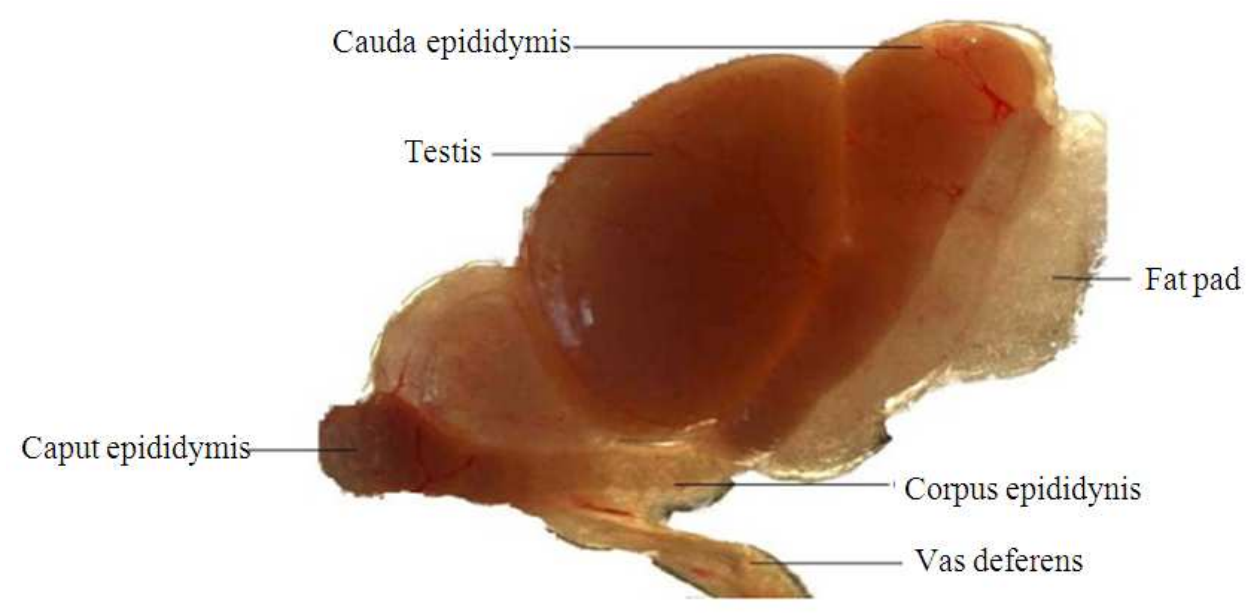

(B)

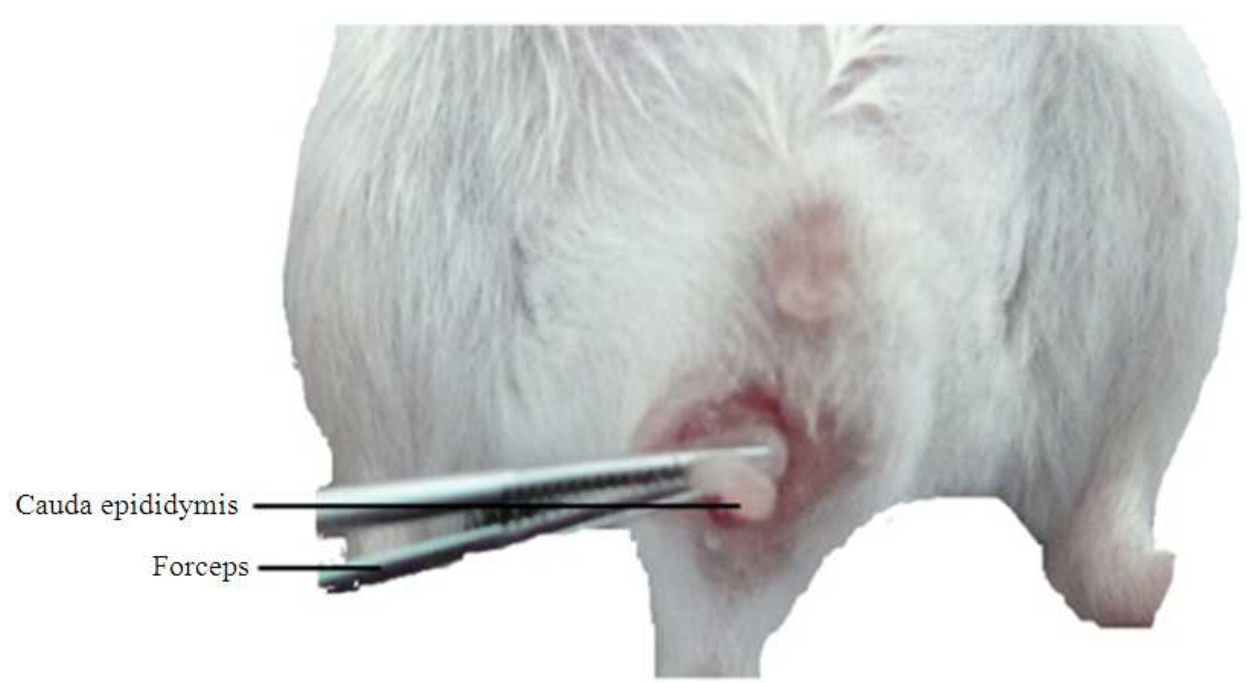

(C)

Fig. 1:A, B, C, D. Illustrates the physiological structure of male mice. C, the part in the forceps is the caudal epididymis 
The tribromoethanol fluid is a compound which was made of $2.5 \mathrm{~g}$ tribromoethanol (Aldrich T4, 840-2, 5mL tertiary amyl alcohol (Aldrich 24, 048-6) and $200 \mathrm{~mL}$ water, while heating and agitating until deliquescenc, then storage at $4^{\circ} \mathrm{C}$ away from light for use. Place the mouse on its back to expose the abdomen and push both testes down into the scrotal sacs by gently applying pressure to the abdomen (Whitting and Wood, 1983). Remove the fur of scrotum, then use a squirt bottle to apply $70 \%$ ethanol to the abdomen and wipe with tissue. Make a $10 \mathrm{~mm}$ incision through the skin along the midline of the scrotal sac (Fig. 1A); locate the midline wall between the testes sacs under the covering membranes of the scrotal sacs. It will appear as a light whitish line. Make a $5 \mathrm{~mm}$ incision in the testes membrane close to the left side of the midline wall. Carefully push the testis to the left.

The vas deferens will appear between the testis and the midline wall as a bright tubule with a single blood vessel that goes down with the vas deferens, caudal epididymis will appear (Fig. 1A and B). Using forceps, pull the caudal epididymis out discreetly (Fig. 1C); collect the sperm and intromission in the Human Tubal Fluid (HTF) medium for In Vitro Fertilization (IVF) while holding the testis in place. Then, cauterize the vestigial caudal epiddymis with tips of forceps made red-hot in flame or cut with fine scissors. Repeat these steps on the other caudal epiddymis. Sew up or clip the skin together with wound clips. At the end of procedure, place the mouse in a clean cage and keep it warm under a $50 \mathrm{~W}$ light bulb (taking care to cover the eyes) or by placing the cage on a warming plate until the mouse recovers from the injected anesthetic. Then, feeding it with no water until $14 \mathrm{~h}$ in case there is wound inflammation and raised it $14 \mathrm{~h}$ later.

\section{RESULTS}

As shown in Fig. 1, make male-sterility mouse by this technique apply to any kinds of male mouse and preliminary studies suggest that comparable results can be obtained with normal method (data not shown). Furthermore, in our hands, an advantage of this procedure is collection of sperm for in vitro fertilization coincides with obtaining male-sterility for other experiment.

As detailed below, we had received some malesterile mice successfully. In addition to providing new methodology for the study of male-sterility, we checked the male-sterility four days after surgery, the experimental male-sterility mouse, wound nearly recovered and has nearly no effect on mate with the pubescent female mouse (Fig. 2A). The wound was traced eight days later, during recovery with no scar (Fig. 2B). Hence, this method proves to provide less trauma to the mouse with healing of wounds faster than normal methods.

This technique may also have other potential applications, for example, the ability to obtain highmating achievement ratio that mate with the pubescent female mouse as he always. We trace the copulation records of the ten experimental male mice before and after surgery and then concluded that the operation has no effect on the mating achievement ratio among the male-sterility (Table 1). These pubescent females (Qing-Yong et al., 2006; Champlin et al., 1973) are mated to vasectomized males or normal males and presence of the vaginal plug indicates mating was successful.

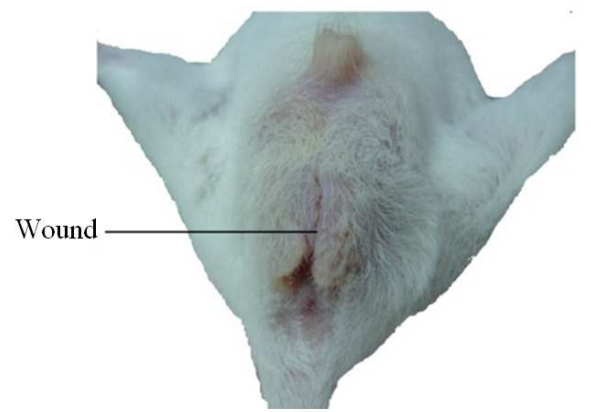

(A)

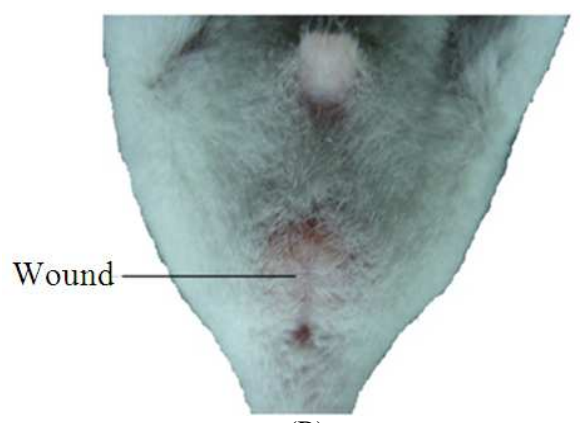

(B)

Fig. 2: Pictures are the healing wound of mouse. A, four days after surgery and the wound nearly healed. $\mathrm{B}$, eight days after surgery and the wound healed already

Table 1: Ten days after surgery, we check the mating success, respectively. Four days later, we repeated the experiment. We collect the records twice and found that there is no significant difference when calculated by $x^{2}$ test $(p>0.05)$

Mating success 10

\begin{tabular}{lll} 
& First time & Second time \\
\hline Male-fertility & 8 & 7 \\
Male -infertility & 7 & 8 \\
\hline
\end{tabular}


American J. Animal \& Vet. Sci., 7 (2): 75-79, 2012

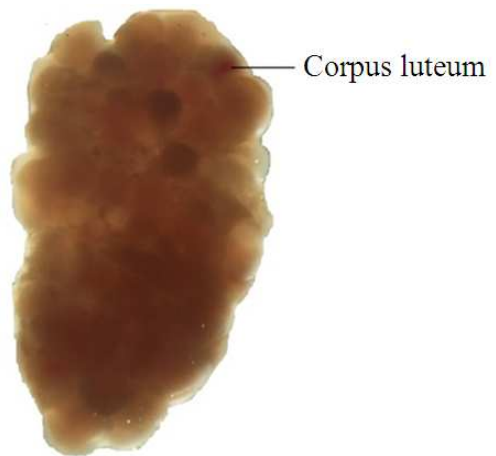

(A)

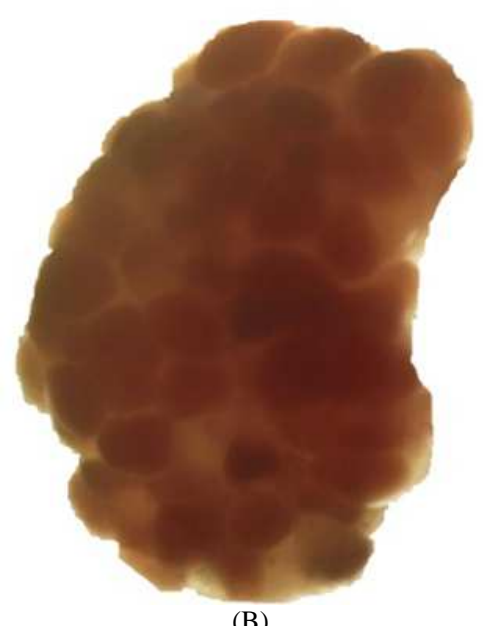

(B)

Fig. 3: Pictures are ovaries taken four days later from the female mouse after successfully mated with male mouse. (A) The ovaries are superscription from female mouse which mate with fertility male mouse successfully four days later. These ovaries are less corpus luteum and still have a little corpus hemorrhagicum, the refractive index of ovaries is good and seem transparent. (B) The ovaries are collected from female mouse mated with infertility male mouse successfully four days later. There are more corpus luteum and little corpus hemorrhagicum, in the ovaries, the refractive index of ovaries was weak.

To certify the operation is successful, we select one female mice random and sacrifice on fourth day which after mating with the experimental male-sterility successfully, in other words, the vaginal plug is seen. The time of the vaginal plug is considered to 0.5 day. One females that were able to mate with fertility males successfully were used as control. We observed the ovaries of the female mouse and found that the female's ovaries are less corpus albicans and still have corpus hemorrhagicum which mated with the experimental fertility male mouse successfully (Fig. 3A), however, the ovaries of the female mouse are number of corpus albicans and have no corpus hemorrhagicum which mated with the experimental male-sterility successfully (Fig. 3B). Further, it seems likely that the technique can be modified to provide a simplified method of preparation of male-sterility for other experiments and reservation of the excellent male mouse.

\section{DISCUSSION}

Compared with both the conventional methods and procedures, resection of the caudal epididymis to make a sterile male mouse is easy to operation and accept, especially for new user. As the materials and method descript, the sterile male mouse is less trauma and cure take shorter time. At fourth day after surgy, the mouse has nearly cured and the wound has hardly any effect on mating with pubescent femal mouse. The wound healing more rapidly. The ratio of mating successfully is not change compare with before surge and the operation is availably (Noormets et al., 2009).

\section{CONCLUSION}

To our knowledge, the progress method to make sterile male mouse combind the two work: get sterile male mouse and reservation of the excellent male nouse and in vitor fertilization to get embryo. In some article, after in vitro fertilization to harvester embryo, the embryo can use as special experimental, such as microinject in any siRNA for knock down some target genes and then in vitro culture to harvester target embryo for transfer or some design experiment.

Disclosure of potential conflicts of interest: The authors indicate no potential conflicts of interest.

\section{ACKNOWLEDGEMENT}

We would like to thank the Animal Nursery of Qing Long Shan of Nanjing for providing male mouse used in these studies. We also thank some schoolmates for their sincere help.

\section{REFERENCES}

Champlin, A.K., D.L. Dorr and A.H. Gates, 1973. Determining the stage of the estrous cycle in the mouse by the appearance of the vagina. Biol. Repord, 8: 491-494. 
Daniel, J.C., 1978. Methods in Mammalian Reproduction. 1st Edn., Academic Press, New York. Academic Press, New York, ISBN-10: 0122018508 pp: 566.

Erhardt, W., A. Hebestedt, G. Aschenbrenner, B. Pichotka and G. Blumel, 1984. A comparative study with various anesthetics in mice (pentobarbitone, ketamine-xylazine, carfentanyl-etomidate). Res. Exp. Med., 184: 159-169. DOI: 10.1007/BF01852390

Nagy, A., 2003. Manipulating the Mouse Embryo: A laboratory Manual. 3rd Edn., Cold Spring Harbor Laboratory Press, New York, ISBN-10: 0879695919, pp: 764.

Noormets, K., S. Kõks, A. Kavak, A. Arend and M. Aunapuu et al., 2009. Male mice with deleted Wolframin (Wfs1) gene have reduced fertility. Reprodu. Biol. Endocrinol., 7: 82-82. DOI: 10.1186/1477-7827-7-82
Nowrouzian, I., H.F. Schels, I. Ghodisan and H. Karimi, 1981. Evaluation of the anaesthetic properties of ketamine and a ketamine/xylazine/atropine combination in sheep. Vet. Rec., 108: 354-356. PMID: 7269183

Papaioannou, V.E. and J.G. Fox, 1993. Efficacy of tribromoethanol anesthesia in mice. Lab. Anim. Sci., 43: 189-192. PMID: 8320967

Qing-Yong, G., J. Zi-jian, L. Xiao-lan, Y. Long and K. Ling, 2006. Research on influences of superovulation in different stage of estrous cycle about mouse. J. Xinjiang Agric. Univ., 29: 16-18.

Whitting, D.G. and M.J. Wood, 1983. Reproductive physiology in the mouse. Biomed. Res. 111: 137164.

Zeller, W., G. Merier, K. Burki and B. Panousis, 1998. Adverse effects of tribromoethanol as used in the production of transgenic mice. Lab, Anim., 32: 407-413. PMID: 9807753 